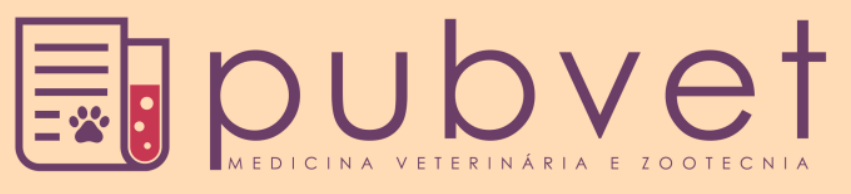

https://doi.org/10.22256/pubvet.v12n3a55.1-10

\title{
Cetoconazol no tratamento e controle das alterações clínicas e hematológicas de hiperadrenocorticismo canino: Relato de Caso
}

\author{
Natália da Costa Sousa ${ }^{1}$, Tiago Montezuma Mendes Pereira ${ }^{\ominus 2}$, Michelle Costa e Silva ${ }^{\bullet}$, \\ Cynthia Levi Baratta Monteiro ${ }^{\ominus} 4$, Antonio Cavalcante Mota Filho ${ }^{\ominus}$, Raimundo Diones \\ Carneiro 06
}

${ }^{I}$ Discente do Curso de Medicina Veterinária - Faculdade Terra Nordeste (FATENE) - E-mail: nataliacostalp@gmail.com

${ }^{2}$ Médico Veterinário, Fortaleza, Ceará, Brasil-E-mail: edmontezuma@hotmail.com

${ }^{3}$ Professora da Faculdade Terra Nordeste (FATENE), Departamento de Medicina Veterinária. Caucaia-CE Brasil-E-mail: michelle.silva@fatene.edu.br ${ }^{4}$ Professora da Faculdade Terra Nordeste (FATENE), Departamento de Medicina Veterinária. Caucaia-CE Brasil-E-mail: cyntia.baratta@fatene.edu.br ${ }^{5}$ Professor da Faculdade Terra Nordeste (FATENE), Departamento de Medicina Veterinária. Caucaia-CE Brasil-E-mail: antonio.mota@fatene.edu.br ${ }^{6}$ Médico Veterinário da Policlínica Veterinária Metropolitana de Caucaia (Fortaleza, Ceará, Brasil)-E-mail: diones.carneiro@fatene.edu.br *Autor para correspondência

RESUMO. O hiperadrenocorticismo (HAC), conhecido também como Síndrome de Cushing, é uma enfermidade que se manifesta por uma série de alterações clinicas e anormalidades multissistêmicas resultantes da maior exposição crônica a glicocorticóides secretados pelas adrenais ou administração excessiva deste hormônio. Duas diferentes categorias de medicamentos são usadas atualmente no tratamento do HAC: inibidores da esteroidogênese e moduladores da secreção de adrenocorticotrófico. Entre os fármacos inibidores de esteroidogênese o mais indicado na prática pelos médicos veterinários é o trilostano. No entanto, devido seu alto custo há a necessidade de se estudar fármacos que possam ter ação sistêmica no controle das alterações clínicas e hematológicas do HAC. Desta forma, o presente trabalho tem como objetivo avaliar o uso de cetoconazol como fármaco de eleição no tratamento e controle das alterações clínicas e hematológicas do hiperadrenocorticismo canino. Foi atendida na Policlínica Veterinária Metropolitana de Caucaia - HVM uma cadela, da raça Poodle, com 8 anos de idade, com diagnóstico de hiperadrenocorticismo. A tutora relatou que a cadela estava a 4 meses sem fazer uso do Trilostano por não ter condições financeiras de manter o tratamento, o que piorou significativamente os sinais clínicos anteriormente apresentados: polidipsia, poliúria, polifagia, pele hiperpigmentada e com lesões, alopecia simétrica, telangiectasia, abdome pendular, fraqueza muscular nas regiões dos membros pélvicos. Constatou-se visualmente a dispneia e na ausculta cardíaca foi detectada taquicardia. Foram então solicitados exames laboratoriais, ultrassonográficos e avaliação cardiológica (eletrocardiograma e ecocardiograma). Diante das alterações nos resultados dos exames o médico veterinário sugeriu a utilização de Cetoconazol com a dose inicial de $5 \mathrm{mg} / \mathrm{kg} / \mathrm{VO} / \mathrm{BID}$ por 7 dias e silimarina $180 \mathrm{mg} / \mathrm{VO} / \mathrm{SID}$. Com a finalidade de controlar os sintomas clínicos da paciente e as alterações observadas nos exames realizados. Após esse período foi observada melhora significativa quanto às alterações hematológicas e bioquímicas da paciente, no entanto clinicamente o animal apresentou piora, apresentando vômitos frequentes e inapetência. Diante do exposto, pode-se concluir que o uso de cetoconazol no tratamento de pacientes com HAC canino deve ser utilizado apenas como tratamento adjuvante, visto que apesar de apresentar melhora significativa das alterações hematológicas e bioquímicas relacionadas ao distúrbio endócrino não proporciona melhora dos sintomas clínicos.

Palavras chave: endocrinopatias, alopecia 


\title{
Use of ketoconazole in the treatment and control of clinical and hematological changes in canine hyperadrenocorticism: Case Report
}

\begin{abstract}
Hyperadrenocorticism (HAC), also known as Cushing's Syndrome, is a disease manifested by a series of clinical changes and multisystem abnormalities resulting from increased chronic exposure to glucocorticoids secreted by the adrenal glands or excessive administration of this hormone. Two different categories of medications are currently used in the treatment of HAC: inhibitors of steroidogenesis and modulators of $\mathrm{ACTH}$ secretion. Among the drugs inhibiting steroidogenesis the most indicated in practice by veterinarians is trilostane. However, due to its high cost, there is a need to study drugs that may have a systemic action in the control of clinical and hematological alterations in $\mathrm{CAH}$. Thus, the present study aims to evaluate the use of ketoconazole as the drug of choice in the treatment and control of clinical and hematological changes of canine hyperadrenocorticism. At 8-year-old Poodle dog with diagnosis of hyperadrenocorticism was attended at the Metropolitan Veterinary Polytechnic of Caucaia - HVM. The guardian reported that the dog was 4 months without using Trilostan because it did not have financial conditions to maintain the treatment, which significantly worsened the clinical signs previously presented: polydipsia, polyuria, polyphagia, hyperpigmented skin with lesions, symmetrical alopecia, telangiectasia, pendular abdomen, muscle weakness in the pelvic limbs. Dyspnea was visually detected and cardiac auscultation detected tachycardia. Laboratory, ultrasonographic and cardiac evaluation (electrocardiogram and echocardiogram) were requested. Due to changes in the results of the tests, the veterinarian suggested the use of ketoconazole at the initial dose of $5 \mathrm{mg} / \mathrm{kg} / \mathrm{VO} / \mathrm{BID}$ for 7 days and silymarin 180mg / VO / SID.com for purpose of controlling the patient's clinical symptoms and the observed changes in the examinations carried out. After this period, there was a significant improvement in the hematological and biochemical alterations of the patient, however the animal clinically presented worsening, presenting frequent vomiting and inappetence. In view of the above, it can be concluded that the use of ketoconazole in the treatment of patients with canine $\mathrm{CAH}$ should be used only as an adjuvant treatment, since despite the significant improvement of the hematological and biochemical changes related to the endocrine disorder does not improve clinical symptoms.
\end{abstract}

Keywords: endocrinopathies, alopecia

\section{Ketoconazol en el tratamiento y control de las alteraciones clínicas y hematológicas de hiperadrenocorticismo canino: Reporte de un caso}

RESUMEN. El hiperadrenocorticismo (HAC), conocido también como Síndrome de Cushing, es una enfermedad que se manifiesta por una serie de alteraciones clínicas y anormalidades multisistémicas resultantes de la mayor exposición crónica a glucocorticoides secretados por la corteza de la glándula adrenal o la administración excesiva de esta hormona. Dos diferentes categorías de medicamentos se utilizan actualmente en el tratamiento del HAC: inhibidores de la esteroidogénesis y moduladores de la secreción de adrenocorticotrófico ACTH. Entre los fármacos inhibidores de esteroidogénesis el más indicado en la práctica por los médicos veterinarios es el trilostán. Sin embargo, debido a su alto costo existe la necesidad de estudiar fármacos que puedan tener acción sistémica en el control de las alteraciones clínicas y hematológicas del HAC. De esta forma, el presente trabajo tiene como objetivo evaluar el uso de ketoconazol como fármaco de elección en el tratamiento y control de las alteraciones clínicas y hematológicas del hiperadrenocorticismo canino. Fue atendida en la Policlínica Veterinaria Metropolitana de Caucaia - HVM una perra, de la raza Poodle, con 8 años de edad, con diagnóstico de hiperadrenocorticismo. La tutora relató que la perra llevaba 4 meses sin hacer uso del Trilostano por no tener condiciones financieras de mantener el tratamiento, lo que empeoró significativamente los signos clínicos anteriormente presentados: polidipsia, poliuria, 
polifagia, piel hiperpigmentada y con lesiones, alopecia simétrica, telangiectasia, abdomen pendular, debilidad muscular en las regiones de los miembros pélvicos. Se constató visualmente la disnea y en la auscultación cardíaca se detectó taquicardia. Se solicitaron exámenes de laboratorio, ultrasonográficos y evaluación cardiológica (electrocardiograma y ecocardiograma). Ante la alteración de los resultados de los exámenes, el médico veterinario sugirió el uso de ketoconazol con la dosis inicial de $5 \mathrm{mg} / \mathrm{kg} / \mathrm{VO} / \mathrm{BID}$ durante 7 días y silimarina $180 \mathrm{mg}$ / VO / SID. Con la finalidad de controlar los síntomas clínicos de la paciente y cambios observados en los exámenes realizados. Después de ese período se observó una mejora significativa en cuanto a las alteraciones hematológicas y bioquímicas de la paciente, sin embargo clínicamente el animal presentó empeoramiento, presentando vómitos frecuentes e inapetencia. En el presente estudio, se puede concluir que el uso de ketoconazol en el tratamiento de pacientes con HAC canino debe ser utilizado sólo como tratamiento adyuvante, ya que a pesar de presentar una mejora significativa de las alteraciones hematológicas y bioquímicas relacionadas al disturbio endocrino no proporciona mejoría de los síntomas clínicos.

Palabras clave: endocrinopatía, alopecia

\section{Introdução}

Os distúrbios endócrinos parecem estar envolvidos em cerca de 10 a $20 \%$ das doenças relatadas em cães e menos de $5 \%$ em gatos. Entre as endocrinopatias que acometem os cães, o hiperadrenocorticismo (HAC) é uma doença relativamente comum, quando comparados aos gatos atendidos na rotina clínica médica de pequenos animais (Engelking, 2010, Nelson \& Couto, 2015).

O HAC, conhecido também como Síndrome de Cushing, é uma enfermidade em que se manifesta uma série de alterações clinicas e anormalidades multissistêmicas resultantes da maior exposição crônica a glicocorticoides secretados pelas adrenais ou administração excessiva deste hormônio (Peterson, 2007). A síndrome pode ser classificada como dependente de hipófise (HAHD), ocorrendo de forma espontânea por um tumor funcional de origem hipofisária o qual secreta hormônio adrenocorticotrófico (ACTH) correspondendo a $80-85 \%$ dos casos, além deste, podem ser classificados como neoplasia adrenocortical dependente de adrenal (HAC-AD) correspondendo à $20 \%$ dos casos e iatrogênica, resultante da administração exógena excessiva ou prolongada de glicocorticoide (Peterson, 2007, Alenza, 2011, Nelson \& Couto, 2015).

Segundo os autores Feldman (1997) e Nelson \& Couto (2015), o hiperadrenocorticismo acomete em geral cães de meia idade a idosos. Considerase que quase todos os cães com a síndrome tenham mais de seis anos de idade. Todas as raças são suscetíveis ao desenvolvimento de HAC, porém animais das raças Poodle, Dachshund, pequenos Terriers, Pastor Alemão, Beagle e Labrador
Retriever são mais predispostas. No que diz respeito à distribuição sexual, alguns autores relatam que não existe predisposição sexual, enquanto que alguns estudos concluem que as fêmeas parecem estar mais predispostas para desenvolver a doença, em destaque o HAC-AD (Peterson, 2007, Alenza, 2011).

A apresentação clínica do hiperadrenocorticismo pode ser variado, de evolução lenta ou de forma insidiosa, mas geralmente os cães acometidos desenvolvem uma clássica combinação de sinais clínicos que incluem polidpsia, poliúria, polifagia, aumento de volume abdominal, dispneia, alterações dermatológicas, fraqueza muscular, dentre outras alterações sistêmicas, bem como anormalidades hematológicas e bioquímicas. Durante a evolução do HAC podem ocorrer várias complicações concomitantes, favorecendo o desenvolvimento de lesões nos órgãos vitais.

A abordagem utilizada para esclarecer ou definir o diagnóstico HAC deve envolver etapas sequencias como: achados do histórico clínico e exame físico que possam sugerir a doença; obtenção dos resultados de exames laboratoriais de rotina que possam ser caraterísticos da suspeita clínica; exames imagiológicos e por fim, testes de exploração funcional endócrina com baixa e alta dose de dexametasona (TSBDD), estimulação do hormônio adrenocorticotrófico (ACTH) e razão urinária cortisol/creatinina (RC:CU) (Nelson \& Couto, 2015, Thrall, 2015).

Para selecionar o tratamento mais adequado para cada paciente em particular deve- se considerar o estado geral do paciente, as características da opção terapêutica, o tipo de 
HAC e a colaboração do proprietário, pois, o objetivo do tratamento é a melhora clínica sem a progressão da doença, evitando suas complicações (Hammerschimidt, 2017). Duas diferentes categorias de medicamentos são usadas atualmente no tratamento do HC: inibidores da esteroidogênese e moduladores da secreção de ACTH. Entre os fármacos inibidores de esteroidogênese o mais indicado na prática pelos médicos veterinários é o trilostano. Devido ao alto custo de manter o tratamento com trilostano os proprietários são obrigados a descontinuar a terapia, fato este que inviabiliza o tratamento correto e a obtenção de melhor prognóstico. Diante disso, surge a necessidade de testar outros fármacos para o controle do distúrbio endócrino, sendo o cetoconazol uma opção com ação semelhante ao trilostano e com um custo mais acessível. Sendo assim, este trabalho teve como objetivo avaliar a ação do cetoconazol como fármaco de eleição no tratamento e controle das alterações clínicas e hematológicas em paciente canino com hiperadrenocorticismo.

\section{Material e Métodos}

Foi atendida na Policlínica Veterinária Metropolitana de Caucaia - HVM uma cadela, da raça Poodle, com 8 anos de idade, pesando 7,85 $\mathrm{kg}$, com histórico de diagnóstico de hiperadrenocorticismo. Durante a anamnese a proprietária relatou que por não ter condições financeiras de manter o tratamento com trilostano sugerido como terapia anteriormente, devido seu alto custo, a cadela estava a 4 meses sem fazer uso desse fármaco e que piorou significativamente dos sinais clínicos anteriormente apresentados: polidpsia, poliúria, polifagia e lesões na pele. No exame físico, notou-se pele muito fina $\mathrm{e}$ hiperpigmentada, alopecia simétrica (Figura $1 \mathrm{~A} \mathrm{e}$ B), telangiectasia, abdome pendular, definhamento muscular nas regiões dos membros pélvicos e como consequência fraqueza muscular (Figura $1 \mathrm{C}$ e D). Constatou-se visualmente a dispneia e na ausculta cardíaca foi detectada taquicardia. Ainda no exame físico se observou que as mucosas se apresentavam normocoradas e a temperatura encontrava-se $39,8^{\circ} \mathrm{C}$.

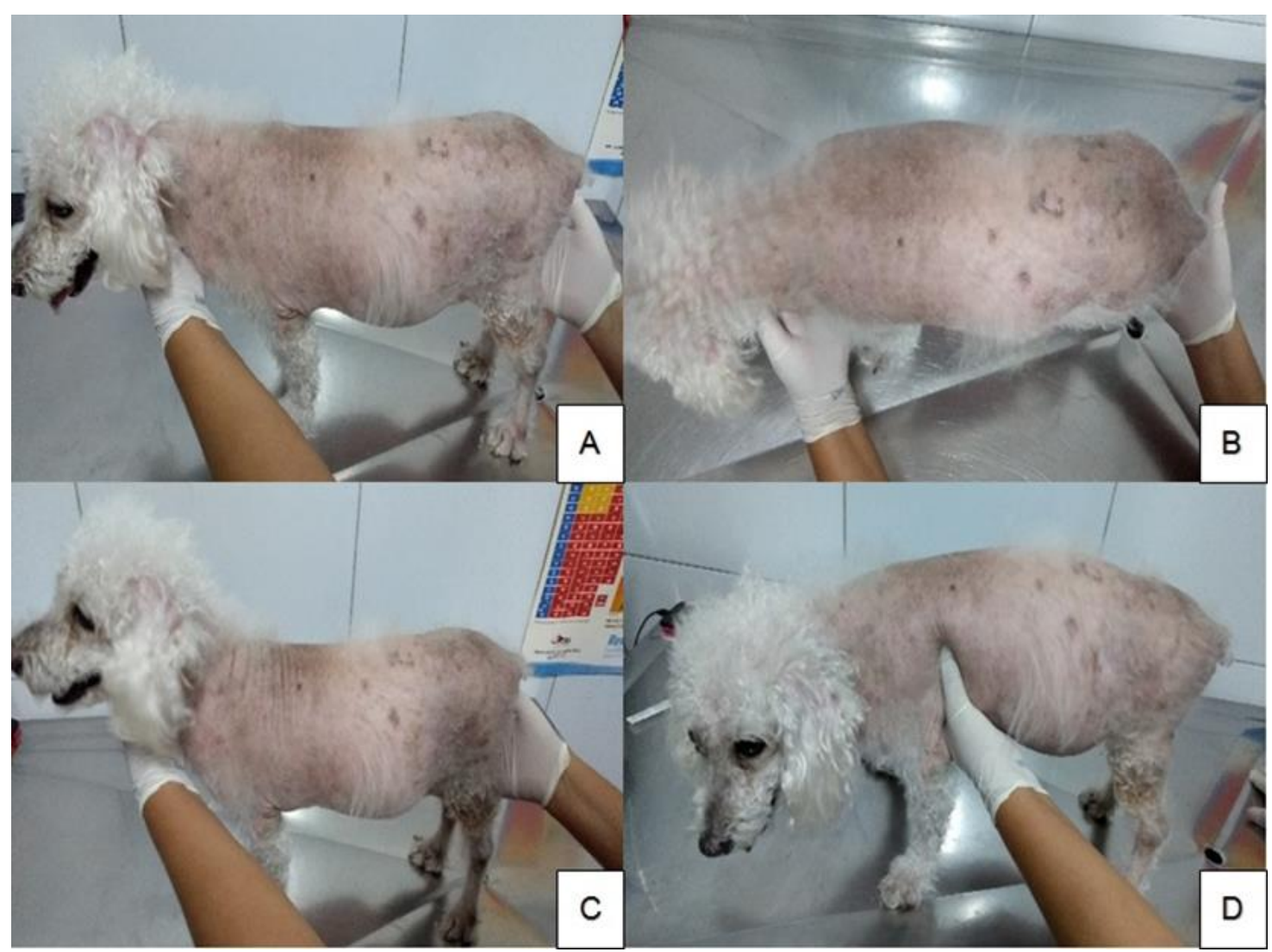

Figura 1. Cadela, poodle diagnosticada com HAC com 4 meses sem tratamento com trilostano apresentando sinais descompensados de HAC. A: pele fina e hiperpgmentação. B: alopecia simétrica. C: abdome pendular. D: definhamento muscular dos membros pélvicos. Fonte: Policlínica Veterinária Metropollitana de Caucaia - HVM. 
Diante da piora do quadro clínico foram realizados hemograma completo, análises bioquímicas (alanina aminotransferase (ALT), fosfatase alcalina (FA), triglicerídeos, colesterol, glicose, creatinina) e exame ultrassonográfico.

\section{Resultados e Discussão}

Os cães acometidos por HAC geralmente desenvolvem uma clássica combinação de sinais clínicos (polidpsia, poliúria, polifagia e lesões na pele) associados ao aumento das concentrações de glicocorticoides (Peterson, 2007), conforme visto na condição clínica na paciente em questão. No que diz respeito ao perfil hematológico, observouse que o animal apresentava na série vermelha (eritrograma) uma trombocitose. Alguns autores afirmam que a contagem de plaquetas eventualmente também pode se encontrar elevada nos casos de HAC. Tais resultados ocorrem devido à ação dos glicocorticoides sobre a medula óssea. $\mathrm{Na}$ série leucocitária os valores referenciaram apenas uma leucocitose com neutrofilia madura (Tabela 1). Na literatura tem sido proposto que a produção excessiva de cortisol se tem como resposta um leucograma de estresse caracterizado por leucocitose, neutrofilia madura pela diminuição do egresso dos neutrófilos da vasculatura e ao aumento da liberação dos neutrófilos da reserva de armazenamento da medula óssea; linfopenia ocorre principalmente pela linfólise dos linfócitos $\mathrm{T}$ sensíveis a esteroides; eosinopenia porque os glicocorticoides diminuem a sua liberação medular (Thrall, 2015, Nelson \& Couto, 2015).

Tabela 1. Resultado do primeiro hemograma realizado em 16/08/2017 no Laboratório de Patologia Clínica da Policlínica Veterinária Metropolitana-HVM.

\begin{tabular}{|c|c|c|c|c|}
\hline Eritrograma & \multicolumn{2}{|c|}{ Valores Encontrados } & \multicolumn{2}{|c|}{ Valores Referenciais } \\
\hline Eritrócitos $\left(/ \mathrm{mm}^{3}\right)$ & \multicolumn{2}{|c|}{5.800 .000} & \multicolumn{2}{|c|}{$5.500 .000-85.000 .000$} \\
\hline Hemoglobina (g/dL) & \multicolumn{2}{|c|}{13.0} & \multicolumn{2}{|c|}{$12-18$} \\
\hline Hematócrito (\%) & \multicolumn{2}{|c|}{39} & \multicolumn{2}{|c|}{$37-55$} \\
\hline VGM (Fl) & \multicolumn{2}{|c|}{67.2} & \multicolumn{2}{|c|}{$60-77$} \\
\hline CHGM (\%) & \multicolumn{2}{|c|}{33,3} & \multicolumn{2}{|c|}{$32-36$} \\
\hline Plaquetas $\left(/ \mathrm{mm}^{3}\right)$ & \multicolumn{2}{|c|}{640.000} & \multicolumn{2}{|c|}{$200.000-500.000$} \\
\hline Proteína Total (g/dL) & \multicolumn{2}{|c|}{7,0} & \multicolumn{2}{|c|}{$6.0-8.0$} \\
\hline \multirow[t]{2}{*}{ Leucograma } & \multicolumn{2}{|c|}{ Valores Encontrados } & \multicolumn{2}{|c|}{ Valores Referenciais } \\
\hline & $\%$ & $/ \mathrm{mm}^{3}$ & $\%$ & Absoluto \\
\hline Leucócitos & & 39.100 & 6.000 & 17.000 \\
\hline Mielócitos & & & 0 & 0 \\
\hline Metamielócitos & & & 0 & 0 \\
\hline Bastões & & & $0-03$ & $0-300$ \\
\hline Segmentados & 94 & 36.754 & $60-77$ & $300-11.500$ \\
\hline Linfócitos & 4 & 1.564 & $12-30$ & $1.000-4.800$ \\
\hline Monócitos & 2 & 782 & $03-10$ & $150-1.350$ \\
\hline
\end{tabular}

Fonte: Laboratório de Patologia Clínica da Policlínica Veterinária Metropolitana - HVM (2017).

As análises bioquímicas apresentaram relevância para o diagnóstico de hiperadrenocorticismo (Tabela 2). O ALT, que apresentou atividade aumentada significativa no referido paciente, é encontrado no citosol dos hepatócitos e liberado sempre que ocorre lesão da membrana hepatocelular. No hiperadrenocorticismo esta elevação é decorrente de acúmulo de glicogênio no hepatócito e esteatose hepática (Gonzalez \& Silva, 2006, Jericó et al., 2015). Quanto ao aumento dos níveis de fosfatase alcalina, este resultado era esperado, visto que mais de $90 \%$ dos cães com HAC apresentam aumento da atividade desta enzima, o qual deve-se a uma isoenzima induzida por esteroide espécie-específica. Essa determinação da isoenzima de FA induzida por glicocorticoide é tão sensível no diagnóstico de hiperadrenocorticismo quanto a mensuração de FA total, mas é não mais específica. O motivo é que a isoenzima induzida por glicocorticoide pode estar aumenta em outras enfermidades, inclusive nas hepatopatias primárias (Thrall, 2015, Nelson \& Couto, 2015). 
Tabela 2. Resultado das primeiras análises bioquímicas realizadas em 16/08/2017 no Laboratório de Patologia Clínica da Policlínica Veterinária Metropolitana - HVM.

\begin{tabular}{lcc}
\hline Bioquímicos & Resultado & Referência \\
\hline ALT (U/L) & 485 & $<50$ \\
Colesterol (mg/dL) & 198 & $135-270$ \\
Fosfatase alcalina (U/L) & 442 & $20-156$ \\
Triglicérides (mg/dL) & 176 & $20-112$ \\
Glicose (mg/dL) & 128 & $70-110$ \\
Creatinina (mg/dL) & 1,4 & $0.5-1.5$ \\
\hline
\end{tabular}

Fonte. Laboratório de Patologia Clínica da Policlínica Veterinária Metropolitana - HVM (2017).

Apesar da literatura consultada descrever que mais de $75 \%$ dos cães com HAC apresentam hipercolesterolemia e/ou hipertrigliceridemia devido à estimulação da lipólise pelos glicocorticóides (Alenza, 2011, Jericó et al., 2015), foi observado apenas um aumento nos triglicerídeos e o colesterol se manteve nos valores de referência. A discreta hiperglicemia observada deve ter sido resultado do aumento da gliconeogênese hepática e do antagonismo à ação da insulina exercido pelos glicocorticoides. Considera-se a possibilidade de diabetes melito concomitante quando o valor da glicose sanguínea for superior a $300 \mathrm{mg} / \mathrm{dL}$ (Thrall, 2015). Os resultados revelaram creatinina dentro dos valores de referência. A creatinina é o metabólito mais específico de diagnóstico de função renal alterada já que não se afeta nem pela dieta nem pelo catabolismo proteico e aumenta pouco em casos de desidratação ou falha cardíaca, a não ser em casos severos (Gonzalez \& Silva, 2006).
A ultrassonografia abdominal foi solicitada com o objetivo de avaliar o tamanho e o formato das adrenais, assim como buscar outras anormalidades nos demais órgãos (Nelson \& Couto, 2015). O aspecto ultrassonográfico das adrenais evidenciou simetria e com dimensões importantemente aumentadas (esquerda $2,34 \mathrm{~cm}$ de comprimento x $1,05 \mathrm{~cm}$ pólo caudal/direita: $2,47 \mathrm{~cm}$ de comprimento $\times 1,04 \mathrm{~cm}$ polo caudal), (Figura 2 A e B), apresentam-se hipoecogênicas, homogenia de formato habitual e contornos regulares características compatíveis com hiperadrenocorticismo. De acordo com (Nelson \& Couto, 2015), os achados de adrenais bilateralmente simétricas de tamanho normal ou grande em cão com hiperadrenocorticismo é uma evidencia de hiperplasia da adrenal causada por HA-HD. O baço apresentou dimensões muito aumentadas (esplenomegalia) com aspecto ultrassonográfico compatível com fibrose ou mineralização.

$\mathrm{Na}$ avaliação da estrutura renal, os rins encontravam-se simétricos, ambos com manutenção da arquitetura córtico medular e perda importante da proporção córtico medular devido ao aumento da espessura da cortical, acompanhada do grande aumento de sua ecogenicidade, podendo assim ser sugestivo de uma nefropatia, sendo essa avaliação dada pela ultrassonografia. Em ambos os rins, observou-se áreas hiperecogenicas, de contornos regulares, formadoras de grande sombra acústica posterior sugerindo microcálculos ou área de mineralização. Em pacientes com hiperadrenocorticismo pode-se notar também mineralização em tecidos moles incluindo a pelve renal.

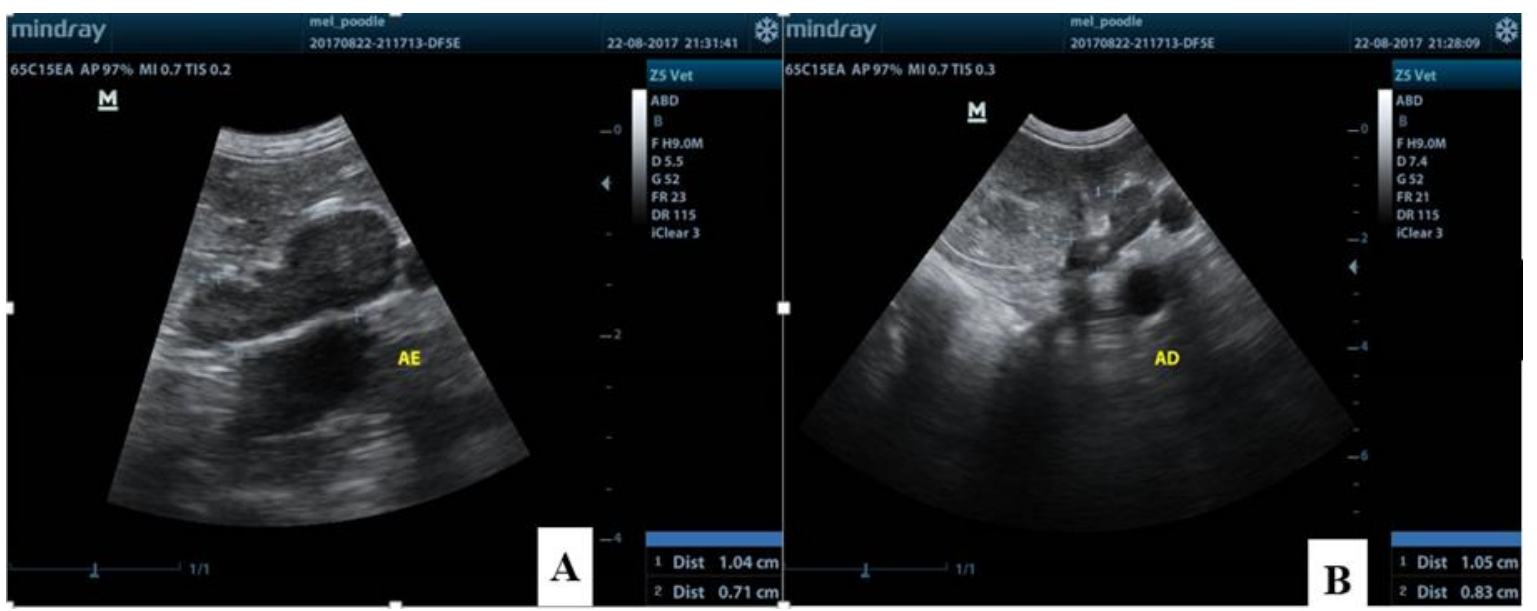

Figura 2. Sonograma das glândulas adrenais, evidenciando simeria e aumento relevante das dimensões. (A) Glândula adrenal esquerda $2,34 \mathrm{~cm}$ de comprimento x $1,05 \mathrm{~cm}$ pólo caudal. (B) Glândula direita $2,47 \mathrm{~cm}$ de comprimento x $1,04 \mathrm{~cm}$ polo caudal. Fonte: Policlínica Veterinária Metropollitana de Caucaia - HVM. 
Também foi relatada hepatomegalia moderada, a qual é bem comum em pacientes com HAC, onde esta alteração pode ocorrer devido ao aumento da síntese de glicogênio e subsequente armazenamento no fígado (Reece \& Penteado Júnior, 1996). A vesícula biliar apresentou-se distendida por conteúdo hipoecogênico (lama biliar) na porção pendente do lúmen. Uma borda contínua e irregular, hipoecogênica a anecóica pode ser visibilizada na periferia interna da vesícula (mucocele). A literatura descreve que a mucocele também está associada à hiperlipidemia e ao HAC (Reece \& Penteado Júnior, 1996, Mesich et al., 2009).

As alterações vistas no estômago foram paredes muito espessas e com perda de definição da estratificação das camadas normoespessas. Características compatíveis com processo inflamatório.

O tratamento instituído para a paciente foi o cetoconazol com a dose inicial de 5 $\mathrm{mg} / \mathrm{kg} / \mathrm{VO} / \mathrm{BID}$ por 7 dias e silimarina 180 $\mathrm{mg} / \mathrm{VO} / \mathrm{SID}$ até novas avaliações. O cetoconazol é um composto imidazol utilizado principalmente como agente antifúngico, no entanto, em doses maiores que as recomendadas para a atividade antifúngica tem ação semelhante àquele do trilostano, inibindo a síntese de esteroides de forma reversível. Tem sido utilizado com sucesso no controle de hiperadrenocorticismo em cães, mas induz mais efeitos colaterais que o trilostano. (Maddison et al., 2011). O protocolo sugerido na literatura é a dose inicial é de $5 \mathrm{mg} / \mathrm{kg} / \mathrm{VO} / \mathrm{BID}$ por 7 dias e, se nenhum efeito adverso for notado, aumentar a dose para $10 \mathrm{mg} / \mathrm{kg} / \mathrm{VO} / \mathrm{BID}$ por 14 dias e avaliar a supressão adrenal com teste de estimulação de ACTH ao final deste período. O uso fica estrito a casos refratários ou de intolerância ao mitotano e ao trilostano ou a condições financeiras inadequadas para proporcionar o tratamento com um desses fármacos. (Maddison et al., 2011, Jericó et al., 2015).

Após os 7 dias de tratamento com cetoconazol a proprietária retornou à consulta com a paciente para novas avaliações e relatou que durante o período de tratamento o animal já apresentava sinais de recuperação, encontrava-se mais ativa e já conseguia se manter em pé, porém não observou melhora em relação a polifagia, polidpsia e poliúria. Passados 14 dias de tratamento, para acompanhamento da progressão clínica foram solicitados novos exames de hemograma completo ALT; aspartato aminotransferase (AST); gama-glutamiltransferase (GGT); FA; triglicerídeos, colesterol, creatinina, sódio e potássio. Ainda, foram realizadas também verificação da pressão arterial sistólica (PAS) e exames cardiológicos de ecocardiograma e eletrocardiograma.

Em comparação dos resultados hematológicos da primeira coleta, podemos notar na série eritrocítica um maior nível de eritrócitos, porém dentro da margem de referência, persistência de trombocitose, com maior nível apresentado. $\mathrm{Na}$ série leucocitária o valor comparado ao primeiro exame teve uma baixa significativa se mantendo dentro dos valores de referência, houve uma diminuição dos neutrófilos, no entanto se manteve elevado (Tabela 3).

No que diz respeito às análises bioquímicas (Tabela 4) os valores de ALT a FA diminuíram pela metade após tratamento, porém continuaram elevados. Os índices de creatinina continuaram dentro dos valores referenciais. Os índices obtidos de colesterol total e triglicérides revelaram hipercolesterolemia e hipertrigliceridemia (Thrall, 2015). O aumento da GGT indica que houve uma lesão hepática, causada pela constante estimulação dos corticosteroides. No entanto, em processos de lesão hepática aguda pode ocorrer aumento imediato da atividade sérica de GGT. O AST se manteve dentro da faixa de limite dos valores de referência. Semelhante com o que acontece com ALT, em cães pode-se verificar aumento discreto da atividade de AST como resultado da indução enzimática por ação de corticosteroides $(\mathrm{O}$ valor observado em relação ao potássio foi um aumento discreto (Barcellos et al., 2011).

A elevação nos níveis de cortisol e aldosterona provoca aumento na reabsorção de sódio (Cook, 2008), elevando seus níveis. Justificando assim o resultado visto no exame da paciente (Tabela 5).

A PAS de $200 \mathrm{mmHg}$ aumentada encontrada no animal com a presença de sinais clínicos evidenciou a hipertensão arterial sistêmica, sendo esta secundária ao hiperadrenocorticismo. A hipertensão sistêmica é constatada em > 50\% dos cães com HAC não tratados o que predispõe a ocorrência de forma secundaria a outros riscos cardiovasculares (Novellas et al., 2008). Os mecanismos primários da hipertensão potencialmente incluem secreção excessiva de renina, ativação do sistema renina-angiotensina, exacerbação da sensibilidade vascular às catecolaminas e redução nos teores de 
prostaglandina vasodilatadoras (Kronenberg, 2009). Os resultados identificados no eletrocardiograma sugeriram sobrecarga de átrio esquerdo na onda $\mathrm{P}$ mitral; sobrecarga de ventrículo esquerdo aumento na duração de QRS; distúrbio eletrolítico infradesnivelamento de segmento ST, esta alteração pode estar associada a hiperpotassemia, que mesmo alterações discretas, como $5,5 \mathrm{mEq} / \mathrm{L}$, podem ser notadas no ECG (Barcellos et al., 2011). O fato de não ter sido feita avaliação eletrolítica completa do paciente em questão, incluindo, por exemplo, dosagem de cálcio, é uma limitação do presente trabalho.

Tabela 3. Resultado do hemograma realizado em 06/09/2017 no Laboratório de Patologia Clínica da Policlínica Veterinária Metropolitana - HVM

\begin{tabular}{|c|c|c|c|c|}
\hline$\overline{\text { Eritrograma }}$ & \multicolumn{2}{|c|}{ Valores Encontrados } & \multicolumn{2}{|c|}{ Valores Referenciais } \\
\hline Eritrócitos $\left(/ \mathrm{mm}^{3}\right)$ & \multicolumn{2}{|c|}{6.200 .000} & \multicolumn{2}{|c|}{$5.500 .000-85.000 .000$} \\
\hline Hemoglobina (g/dL) & \multicolumn{2}{|c|}{14.0} & \multicolumn{2}{|c|}{$12-18$} \\
\hline Hematócrito (\%) & \multicolumn{2}{|c|}{43} & \multicolumn{2}{|c|}{$37-55$} \\
\hline VGM (Fl) & \multicolumn{2}{|c|}{69.3} & \multicolumn{2}{|c|}{$60-77$} \\
\hline CHGM (\%) & \multicolumn{2}{|c|}{33,7} & \multicolumn{2}{|c|}{$32-36$} \\
\hline Plaquetas $\left(/ \mathrm{mm}^{3}\right)$ & \multicolumn{2}{|c|}{760.000} & \multicolumn{2}{|c|}{$200.000-500.000$} \\
\hline Proteína Total $(\mathrm{g} / \mathrm{dL})$ & \multicolumn{2}{|c|}{7.6} & \multicolumn{2}{|c|}{$6.0-8.0$} \\
\hline \multirow[t]{2}{*}{$\overline{\text { Leucograma }}$} & \multicolumn{2}{|c|}{ Valores Encontrados } & \multicolumn{2}{|c|}{ Valores Referenciais } \\
\hline & $\%$ & $/ \mathrm{mm}^{3}$ & $\%$ & Absoluto \\
\hline Leucócitos & & 14.200 & 6.000 & 17000 \\
\hline Mielócitos & & & 0 & 0 \\
\hline Metamielócitos & & & 0 & 0 \\
\hline Bastões & & & $0-03$ & $0-300$ \\
\hline Segmentados & 92 & 13.064 & $60-77$ & $300-11.500$ \\
\hline Linfócitos & 4 & 1.564 & $12-30$ & $1.000-4.800$ \\
\hline Monócitos & 2 & 284 & $03-10$ & $150-1.350$ \\
\hline Observação: Plaquetas Gigantes & & & & \\
\hline
\end{tabular}

Fonte: Laboratório de Patologia Clínica da Policlínica Veterinária Metropolitana - HVM (2017).

As alterações encontradas no ecocardiograma (endocardiose de valva mitral, Insuficiência valvar mitral de grau discreto, insuficiência valvar pulmonar de grau discreto, espessamento discreto de septo interventricular (hipertrofia concêntrica de miocárdio) no animal relatado estão relacionados ao ventrículo esquerdo, assim como em humanos, sugerem o remodelamento miocárdico ventricular esquerdo em virtude da hipertensão arterial sistêmica de origem secundária a HAC (Yiu et al., 2012, Schober et al., 1999), corroborando com os achados do ECG que sugerirão sobrecarga de átrio e ventrículo esquerdo.

Devido a melhora significativa apresentada pelo animal em questão, demonstrando boa tolerância ao medicamento e pelos resultados observados nos exames a dose do cetoconazol foi aumentada para $10 \mathrm{mg} / \mathrm{kg}$ a cada 12 horas durante 14 dias com retorno $15^{\mathrm{a}}$ dia de tratamento. Também foi instituído para o controle da pressão arterial a amlodipina $0,2 \mathrm{mg} / \mathrm{kg}$. A literatura sugere que após 14 dias de tratamento caso não ocorra algum efeito adverso, se faz necessário a supressão adrenal com teste de estimulação do ACTH (Maddison et al., 2011). A questão de não ter sido feito o teste devido a condição financeira atual da proprietária, limitou avaliações hormonais mais especificas. Após a data de início com a dose de $10 \mathrm{mg} / \mathrm{kg}$, com menos de 5 dias, proprietária relata que o animal não passa muito bem, com vômitos constantes e inapetência. Diante do quadro a proprietária suspende as medicações via oral. Após a suspensão do cetoconazol, segunda a tutora, os vômitos foram cessados e voltou a se alimentar normalmente.

Após uma semana sem fazer uso do cetoconazol, optou-se em fazer uma nova tentativa com a dose inicial $5 \mathrm{mg} / \mathrm{kg} / \mathrm{BID}$. Instituiu-se protetor gástrico, omeprazol $10 \mathrm{mg} / \mathrm{SID}$ antes da alimentação, para controle dos vômitos, plasil gotas de uso pediátrico, 8 gotas/BID. Ainda, foi realizado exame de ultrassom.

Em comparação com os achados vistos no primeiro ultrassom foi possível observar a permanência das alterações renais; nas adrenais 
com dimensões importantemente aumentadas (esquerda 2,42 cm de comprimento x $1,08 \mathrm{~cm}$ pólo caudal/direita: $2,37 \mathrm{~cm}$ de comprimento $x 1,03 \mathrm{~cm}$ polo caudal); no baço; no fígado, e estômago. Já a vesícula biliar apresentou menor ecogenicidade quando comparado ao exame anterior.

Tabela 4: Resultado das análises bioquímicas realizadas no dia 06/09/2017 no Laboratório de Patologia Clínica da Policlínica Veterinária Metropolitana - HVM.

\begin{tabular}{lcc}
\hline \multicolumn{1}{c}{ Bioquímicos } & Resultado & Referência \\
\hline ALT (U/L) & 210 & $<50$ \\
AST (U/L) & 66 & $23-66$ \\
GAMA GT (U/L) & 57 & $1.2-8.0$ \\
Colesterol (mg/dL) & 311 & $135-270$ \\
Fosfatase Alcalina U/L & 342 & $20-156$ \\
Triglicérides (mg/dL) & 122 & $20-112$ \\
Creatinina (mg/dL) & 1,4 & $0.5-1.5$
\end{tabular}

Fonte: Laboratório de Patologia Clínica da Policlínica Veterinária Metropolitana - HVM (2017).

Com dois dias de tratamento a proprietária relatou que as crises de vômitos voltaram e logo foi suspendida a medicação. Os efeitos gastrointestinais observados na paciente, incluindo anorexia, vômitos e diarreia são aqueles mais comuns relacionados à administração do cetoconazol (Maddison et al., 2011).

Tabela 5: Resultado laboratorial da análise dos eletrólitos realizados no dia 09.10.2017

\begin{tabular}{lcc}
\hline Eletrólitos & Resultado & Referência \\
\hline Potássio & $5,3 \mathrm{mEq} / \mathrm{L}$ & $3.5-5.0 \mathrm{mEq} / \mathrm{L}$ \\
Sódio & $152,0 \mathrm{mEq} / \mathrm{L}$ & $138-148 \mathrm{mEq} / \mathrm{L}$ \\
\hline
\end{tabular}

Fonte: Pathovet, 2017.

\section{Conclusão}

O Cetoconazol deve ser utilizado apenas como tratamento adjuvante em casos de hiperadrenocorticismo canino, visto que apesar de apresentar melhora significativa das alterações hematológicas e bioquímicas relacionadas ao distúrbio endócrino, não proporciona melhora dos sintomas clínicos.

\section{Referências Bibliográficas}

Alenza, D. P. 2011. Hiperadrenocorticism: Are we over-diagnosing it. Proceedings of the Southern European Conference \& Congreso Nacional AVEPA. Espanha. Barcelona, Espanha.

Barcellos, H. H. A., Melatti, L., Linck, C. M., Gavioli, F. B., Quadros, A. M., Palma, M. D., Casagrande, G., Oro, G., Valle, S. F. \& Motta,
A. C. 2011. Aspectos clínicos e laboratoriais em cães com babesiose crônica. Congresso Brasileiro de Medicina Veterinária. Florianópolis.

Cook, A. K. 2008. Trilostane: A therapeutic consideration for canine hyperadrenocorticism. Veterinary Medicine, 103, 104-116.

Engelking, L. 2010. Fisiologia endócrina $e$ metabólica em medicina veterinária. Roca, São Paulo, Brasil.

Feldman, E. C. 1997. Tratado de medicina interna veterinária.

Gonzalez, F. H. D. \& Silva, S. C. 2006. Introdução à bioquímica clínica animal. Gráfica de Universidade Federal do Rio Grande do Sul, Porto Alegre, Rio Grade do Sul.

Hammerschimidt, J. 2017. O prontuário médico veterinário: requisitos e importância. In: Tostes, R. A., Reis, S. T. J. \& Castilho, V. V. (eds.) Tratado de Medicina Veterinária Legal. Medvep, Curitiba, Paraná, Brasil. .

Jericó, M. M., Kogika, M. M. \& Andrade Neto, J. P. 2015. Tratado de medicina interna de cães e gatos. Guanabara Koogan, Rio de Janeiro, Brasil.

Kronenberg, H. M. 2009. Williams tratado de endocrinologia. Elsevier, Rio de Janeiro, Brasil.

Maddison, J. E., Page, S. W. \& Church, D. B. 2011. Farmacologia clínica de pequenos animais. In: Philip, G. A. \& Thomas, A. F. (eds.) Medicamentos e reprodução. Elsevier Brasil, São Paulo.

Mesich, M. L. L., Mayhew, P. D., Paek, M., Holt, D. E. \& Brown, D. C. 2009. Gall bladder mucoceles and their association with endocrinopathies in dogs: A retrospective case- control study. Journal of Small Animal Practice, 50, 630-635.

Nelson, R. W. \& Couto, C. G. 2015. Medicina interna de pequenos animais. Elsevier Editora, Amsterdan.

Novellas, R., Gopegui, R. R. \& Espada, Y. 2008. Determination of renal vascular resistence ind dog with diabetes mellitus and hyperadrenocorticismo. Veterinary Records 163, 592-596. Veterinary Records, 163, 592596.

Peterson, M. E. 2007. Diagnosis of hyperadrenocorticism in dogs. Clinical Techniques in Small Animal Practice, 22, 2-11. 
Reece, W. O. \& Penteado Júnior, N. 1996. Fisiologia de animais domésticos. Roca, São Paulo.

Schober, K. E., Kirbach, B. \& Oechtering, G. 1999. Noninvasive assessment of myocardial cell injury in dogs with suspected cardiac contusion. Journal of Veterinary Cardiology, $1,17-25$.

Thrall, M. A. 2015. Hematologia e Bioquímica Clínica Veterinária. Editora Roca, São Paulo, Brasil.

Yiu, K. H., Marsan, N. A., Delgado, V., Biermasz, N. R., Holman, E. R., Smit, J. W. A., Feelders,
R. A., Bax, J. J. \& Pereira, A. M. 2012. Increased myocardial fibrosis and left ventricular dysfunction in Cushing's syndrome. European Journal of Endocrinology, 166, 27-34.

Article History:

Received 18 November 2017

Accepted 6 January 2018

Available online 2 March 2018

License information: This is an open-access article distributed under the terms of the Creative Commons Attribution License 4.0, which permits unrestricted use, distribution, and reproduction in any medium, provided the original work is properly cited 